\section{Esclerosis múltiple y embarazo}

\section{Multiple Sclerosis and Pregnancy}

\section{Sr. Editor:}

La esclerosis múltiple (EM) es una enfermedad crónica inflamatoria que afecta en forma predominante a adultos jóvenes, con un promedio de edad de presentación de 30 años y un franco predominio femenino. Los últimos 50 años se ha asistido a un aumento en la incidencia de la enfermedad en mujeres; habiendo aumentado la proporción entre mujeres y hombres de 2,1:1 a 3,5: $1^{1}$. Por constituir esta una población en edad fértil, el tema de las relaciones entre embarazo, esclerosis múltiple y los riesgos y beneficios de la terapia de la enfermedad, tiene especial relevancia tanto para las pacientes como para los médicos².

\section{Relaciones entre embarazo y esclerosis múltiple}

Hasta hace poco tiempo se mantenía la idea de que el embarazo tenía un efecto adverso sobre la EM y se recomendaba evitarlo e inclusive su interrupción. Estudios controlados más recientes han modificado este concepto. Se ha demostrado que la frecuencia de recaídas disminuye durante el segundo y tercer trimestre del embarazo para aumentar después del parto, especialmente en los primeros tres meses. Esta reducción durante el embarazo es de un promedio de $40 \%$, lo que se compara favorablemente con los efectos reportados por las terapias actuales ${ }^{3}$. No hay evidencias de que a largo plazo las pacientes que se han embarazado tengan un curso diferente a las no embarazadas. Publicaciones recientes sugieren más bien un efecto beneficioso a largo plazo. En un grupo de 330 pacientes seguidas durante 18 años se demostró que aquellas madres que han tenido uno o más hijos tienen un curso más benigno, expresado por el tiempo que alcanzan una discapacidad, medida por la dificultad en la deambulación independiente o uso de silla de ruedas ${ }^{4}$. Un estudio reciente en Australia mostró un efecto protector del embarazo sobre la aparición de un primer brote de enfermedad desmielinizante, siendo este efecto acumulativo, dependiendo del número de embarazos $^{5}$. Se ha postulado que el aumento en la edad media del embarazo de 23,2 a 32 años en occidente, se correlaciona entre otros factores, con el aumento de la incidencia de esclerosis múltiple en mujeres.

\section{Efectos de la esclerosis múltiple sobre el embarazo}

En general la esclerosis múltiple no tiene efectos sobre la fertilidad, el embarazo ni el parto. En promedio, la edad de gestación es mayor en pacientes con EM. No hay mayor frecuencia de abortos ni de malformaciones. Sin embargo, parece haber una mayor proporción de partos por cesárea y un peso menor del recién nacido. No se han demostrado efectos adversos a largo plazo en el recién nacido ${ }^{6}$.

La enfermedad no tiene efectos sobre la lactancia. Por otra parte, ningún estudio ha demostrado efectos deletéreos de la lactancia sobre el curso de la esclerosis múltiple. Un estudio reciente afirma que la lactancia estricta reduciría el riesgo de recaídas post parto ${ }^{7}$.

\section{Efecto de drogas modificadoras de la esclerosis múltiple en el embarazo}

La información disponible sobre la seguridad de las drogas aprobadas para el tratamiento de la enfermedad, sobre la fertilidad, embarazo y lactancia aún no es concluyente.

La recomendación general es suspender las drogas antes de la concepción, por su potencial de daño. Sin embargo, no es infrecuente la exposición prenatal, ya que alrededor el $50 \%$ de los embarazos no son planificados. No se recomienda su uso

Tabla 1. Recomendaciones terapéuticas para pacientes con esclerosis múltiple en el embarazo

\section{Planificación del embarazo}

Suspender acetato glatiramer, beta inferferones y natalizumab 2 meses antes

Suspender fingolimod 3 meses antes

Suspender mitoxantrone 6 meses antes

\section{Crisis durante el embarazo}

Metilprednisolona todo el embarazo

Inmunoglobulina en primer trimestre

\section{Post parto}

Metilprednisolona

Inmunoglobulina

\section{Lactancia}

Enfermedad previa poco activa

Lactancia estricta y reiniciar terapia a los 3-6 meses Enfermedad previa activa

Suspender lactancia y reiniciar terapia 
durante el embarazo a pesar de una enfermedad activa, prefiriéndose el uso de pulsos de metilprednisolona o inmunoglobulinas.

Los interferones $1 \mathrm{a}$ y $1 \mathrm{~b}$ no atraviesan la placenta, sin embargo, estarían relativamente contraindicados en mujeres que planeen un embarazo por sus posibles propiedades abortivas. No se sabe si se excretan por la leche materna por lo que su uso en la lactancia no está recomendado. El acetato de glatiramer no atraviesa la barrera placentaria y probablemente es compatible con la lactancia. El anticuerpo monoclonal natalizumab no atraviesa la placenta durante la organogénesis. Sin embargo, la atraviesa en el segundo y tercer trimestre. $\mathrm{Su}$ uso no está recomendado durante el embarazo ni la lactancia. Los datos en humanos muestran que la tasa de abortos y malformaciones son las esperadas para la población general.

Los datos disponibles permiten concluir que las moléculas pequeñas tienen un mayor riesgo, ya que son capaces de atravesar la placenta y son secretadas por la leche materna. En el caso de fingolimod, laquinimod y dimetil fumarato son sospechosas de causar daño, pero aun no hay evidencias concluyentes. Para la mitoxantrona, teriflunomida y cladibrina hay evidencias de riesgo fetal. De acuerdo a la información disponible no hay evidencias para la interrupción del embarazo después de la exposición materna a fármacos que modifican la enfermedad, tal vez con la excepción de la mitoxantrona.

Por el aumento del riesgo de recaídas durante el período post parto se recomienda administrar un curso breve de metilprednisolona o de inmunoglobulina, fármacos que no tienen efectos sobre la lactancia. Una vez ocurrido el parto se plantea la disyuntiva de cuándo reiniciar las terapias inmunomoduladoras. Las opciones son reiniciar la terapia inmediatamente post parto y suprimir la lactancia en pacientes con enfermedad previa activa. En caso contrario parece recomendable lactancia estricta y reiniciar la terapia en un plazo de 3 a 6 meses. Aparentemente el acetato de glatiramer tendría un perfil más alto de seguridad, siendo una opción para la terapia con pacientes con enfermedad agresiva ${ }^{2,8}$.

Fernando Vergara E. Clínica Alemana de Santiago. Facultad de Medicina Universidad de los Andes. Santiago de Chile.

\section{Referencias}

1. Koch-Henriksen N, Sorensen PS. The changing demographic pattern of multiple sclerosis epidemiology. Lancet Neurol 2010; 9: 520-32.

2. Coyle PK. Pregnancy and Multiple Sclerosis. Neurol Clin 2012; 30: 877-88.

3. Vukusic S, Hutchinson M, Hours M, Moreau T, Cortinovis-Tourniaire P, Adeleine P, Confavreux C. Pregnancy and multiple sclerosis (the PRIMS study): clinical predictors of post-partum relapse. Brain 2004, 127 (6), 1353-60.

4. D'hooghe MB, Haentjens P, Nagels G, D'Hooghe T, De Keyser J. Menarche, oral contraceptives, pregnancy and progression of disability in relapsing onset and progressive onset multiple sclerosis. J Neurol 2012, 259: 855-61.

5. Ponsonby AL, Lucas RM, van der Mei IA, Dear K, Valery PC, Pender MP, Taylor BV, et al. Offspring number, pregnancy, and risk of a first clinical demyelinating event: the autoimmune study. Neurology 2012; 78: 967-74.

6. Kelly VM, Nelson LM, Chakravarty EF. Obstetric outcomes in women with multiple sclerosis and epilepsy. Neurology 2009; 73: 1831-6.

7. Langer-Gould A, Huang SM, Gupta R, Leimpeter AD, Greenwood E, Albers KB, et al. Exclusive breastfeeding and the risk of postpartum relapses in women with multiple sclerosis. Arch Neurol 2009; 66: 958-63.

8. Lu E, Wang BW, Guimond C, Synnes A, Sadovnick D, Tremlett H Disease-modifying drugs for multiple sclerosis in pregnancy: a systematic review Disease modifying. Neurology 2012; 79: 1130-1035. 